\title{
Aglle Information Systems
}

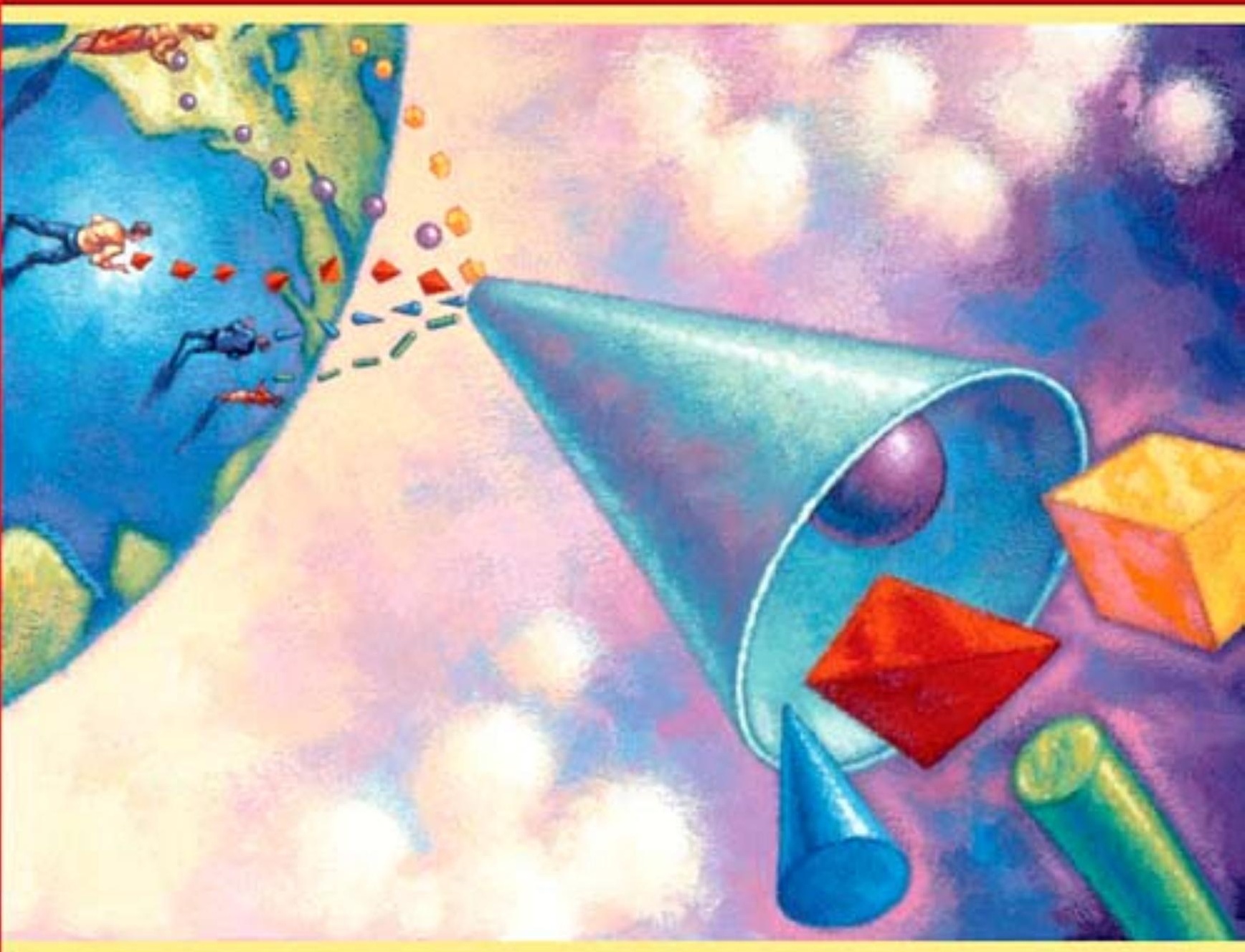

Conceptualization, Construction, and Management

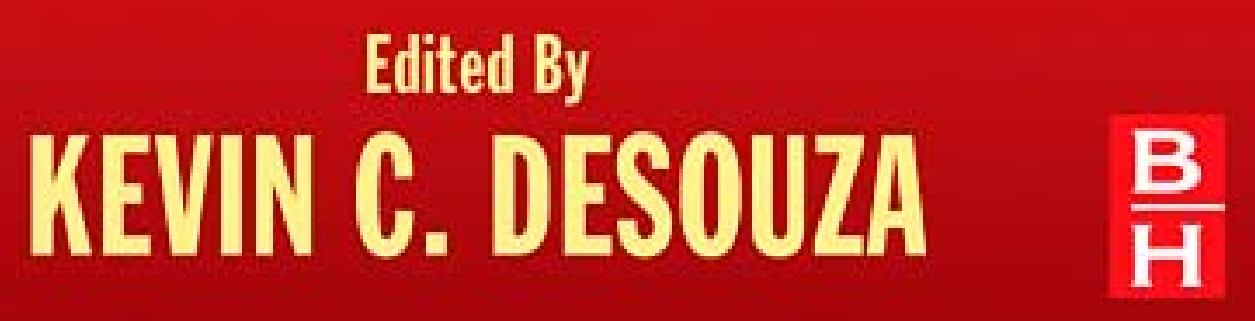




\title{
Business Agility: Need, Readiness and Alignment with IT Strategies $^{1}$
}

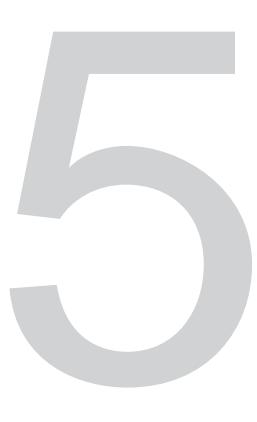

\author{
Marcel van Oosterhout, Eric Waarts, \\ Eric van Heck, and Jos van Hillegersberg
}

The concept of agility originated at the end of the eighties and in the early nineties in the manufacturing area in the United States. Agile Manufacturing was first introduced with the publication of a report entitled 21 $1^{\text {st }}$ Century Manufacturing Enterprise Strategy (Goldman et al., 1991). This was followed by a series of publications on agile manufacturing and agile corporations (Kidd, 1994; Kidd, 1995; Goldman, Nagel, and Preiss, 1995; Dove, 2001). The concept was extended to supply chains and business networks (Christopher, 1992; Mason-Jones and Towill, 1999; Van Hoek, Harrison, and Christopher, 2001; Swafford, 2003; Yusuf, Gunasekaran, Adeleye, and Sivayoganathan, 2004).

Despite the history of the concept, there is by far no consensus yet as to what exactly agility is. Nor is there a consensus on how one could assess and achieve agility (Schrage, 2004). Very few studies have attempted to empirically study the need for agility. What are the contributing factors requiring organizations to be agile, and what is the relative importance of these factors? Furthermore, which of these factors are related to Information Technology (IT) and how does IT enable or hinder the required level of agility?

The central question of this chapter is: What are the contributing factors that require business agility, and what IT strategies can be implemented for enhancing business agility? The sub-questions are:

- How can we define the concept of business agility?

- Do change factors that create a high business agility need to be generic or sector-specific?

- Is there a difference between various industry sectors on the perceived business agility readiness?

${ }^{1}$ The authors gratefully acknowledge the financial support of Hewlett Packard for funding this research. Furthermore, we thank Professor Peter Vervest, Professor Kenneth Preiss, and Dr. Otto Koppius for their comments and feedback, all of the companies for their participation, and our research assistants Roger Carvalho, Rinske Verwaal, Roelof Valkenier, Barbara Hertogs, and Stijn van den Bout for their support in the field research. 
- Are there differences between three domains of business agility (operational, customer, and network) with regard to the need for business agility and the perceived readiness of organizations?

- Which IT strategies can be defined for enhancing business agility, depending on the business agility need and business agility readiness level?

\section{Business Agility}

Even though much has been said and written on agility, a consensus on a definition of agility has not yet emerged. Wadhwa and Rao (2003) describe the differences and overlap between flexibility and agility. Flexibility is defined as a predetermined response to a predictable change, while agility entails an innovative response to an unpredictable change. Flexibility is focused on single systems for low to medium rates of change, while agility is focused on groups of systems to deal with high rates of change. A variety of views on business agility can be found in the literature (Goldman et al., 1995; Sharifi and Zhang, 1999; Dove, 2001; Hooper et al., 2001; Ramasesh et al., 2001; Conboy and Fitzgerald, 2004). The definitions provide some common aspects.

Agility is a way to cope with external and internal changes, which are highly uncertain. Three types of perceived uncertainty can be distinguished: state uncertainty, effect uncertainty, and response uncertainty (Milliken, 1987). State uncertainty relates to unpredictability about whether or when a certain change will happen. Effect uncertainty relates to the inability to predict what the nature of the impact (i.e., effects) of a change will be on the organization. Some changes are quite predictable (e.g., deregulation in the telecom and energy sector); however, often the speed and exact requirements to the organization and processes are quite uncertain. Response uncertainty is defined as a lack of knowledge of response options and/or an inability to predict the likely consequences of a response choice.

Business agility can be implemented either proactively (leading or initiating a change-placing organizations in a leadership position) or reactively (responding to change, either opportunistic or degenerative, in order to retain competitiveness) (Canter, 2000). Dove (2001) highlights the importance of both sensing capabilities (detecting, anticipating) and responding capabilities (physical ability to act rapidly and with relative ease) (Dove, 2001). The concept of quickness and therefore speed is at the heart of agility-it is the capability of an organization to rapidly execute decisionmaking and operational cycles (Canter, 2000). Speed can be required in various areas, like time to market new products, time to process an order or service request, time to assemble a virtual business network for collaboration, time to reconfigure organizational processes and systems to react to certain changes, and so on.

Sambamurthy, Bharadwaj, and Grover (2003) distinguish three interrelated capabilities of agility: operational agility, customer agility, and partnering agility. For each capability, they describe the role and impact of IT. This distinction is in line with types of strategic agility as defined by Weill, Subramani, and Broadbent (2002), who make a distinction between business initiatives aimed at increasing strategic agility based on their position on the value net: demand-side initiatives (customer agility), supply-side initiatives (partnering agility) and internally focused initiatives (operational agility).

Taking all of the above considerations into account, the definition of business agility in this study will be:

Business agility is the ability to sense highly uncertain external and internal changes, and respond to them reactively or proactively, based on innovation of the 
internal operational processes, involving the customer in exploration and exploitation activities, while leveraging the capabilities of partners in the business network.

\section{An Agility Framework}

Building on the work by Sharifi and Zhang (1999) we constructed a framework to analyze business agility in detail (Figure 5.1).

The starting point of our model is the contributing factors, which are external and internal changes that can create a need for business agility (based on Sharifi and Zhang, 1999). In this chapter, we focus on the analyses of change factors, where a required response of the organization is related, directly or indirectly, to the organization's IT capability.

An organization's business agility readiness is determined by its business agility capabilities. Business agility capabilities are the means or barriers for a business to enhance its business agility. Business agility capabilities can be categorized based on the work of Sambamurthy, Bharadwaj, and Grover (2003) and Weill, Subramani, and Broadbent (2002). The business agility capabilities are the reasons behind the existence or nonexistence of agility gaps. If there is a mismatch between the businesses agility need and the business agility readiness, there is a business agility gap. This has implications for the business agility IT strategy.

In this chapter, we will report on the perceived business agility need (BAN) and the perceived business agility readiness (BAR) for external and internal change factors that are directly or indirectly related to the organization's IT. We will also discuss implications for business agility IT strategies to close the business agility gap (BAG).

\section{Methodology}

Based on the literature review, we constructed a questionnaire. We used feedback from experts and two workshops to test and improve the questionnaire. We chose to use multiple methods for data gathering in order to provide a rich description on the

\section{Figure 5.1}

\section{Conceptual Framework*}

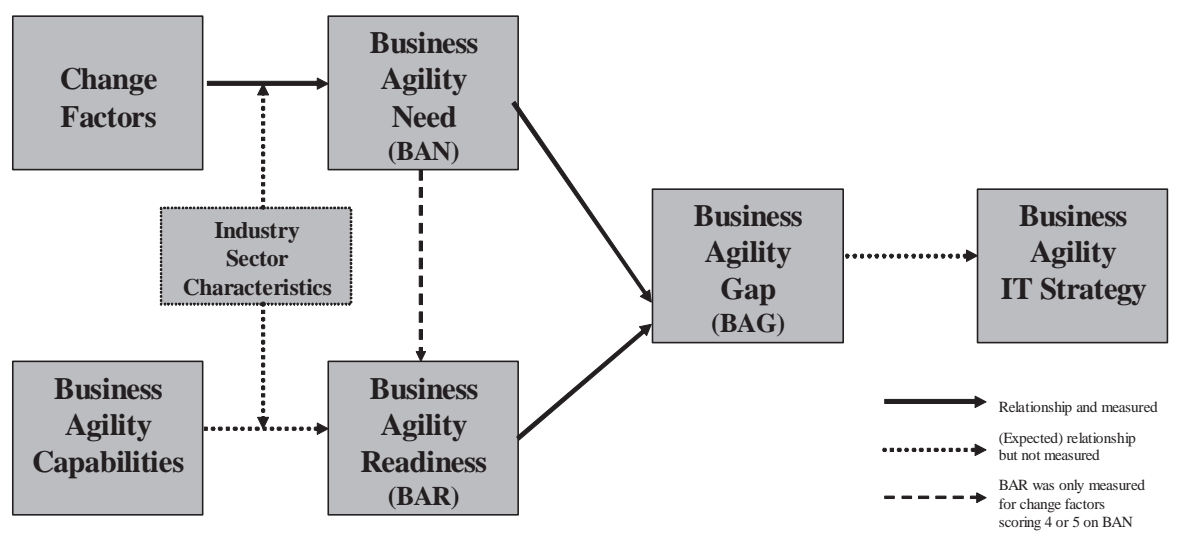

*Adapted from Sharifi and Zhang, 1999. 
topic. We gathered quantitative data via an online questionnaire (110 respondents). This was complemented with in-depth qualitative data, gathered via interviews with 50 managers and workshop discussions. The results were validated by interviews with 14 sector experts.

Based on literature research and workshops with experts, we constructed a questionnaire containing 27 change factors, covering the three agility capabilities of our framework: factors requiring operational agility, factors requiring customer agility, and factors requiring business network and partnering agility. An overview of these change factors can be found in Table 5.1. ${ }^{2}$

Each change factor in the survey had to be scored on a Likert-5 scale. If the perceived BAN due to a certain change factor was high (score 4 or 5), a second question was posed regarding the perceived BAR (also on a Likert-5 scale). The BAG was measured as BAN minus BAR. In the next part of the questionnaire for the 10 change factors with the highest BAG score of the respondent, open questions were generated. For each BAG, the respondent was asked to elaborate on the bottleneck(s) and measures in the required business agility capabilities to deal with the BAG. This way, the questionnaire generated both quantitative as well as qualitative data on agility capabilities as enablers or disablers. We did a cross-check on possible survey fatigue which might bias our results. We found no difference in the variance of answers between the first half of the survey with the second half. Furthermore, we checked the number of responses to individual items in the second half of the questionnaire and compared it with the first half.

For the interviews with managers within each sector, a sample of organizations was selected. Criteria to select organizations were their position in the market (in the business sectors top market share players with considerable size). Within each organization, at least two managers were asked to fill out the survey, as a basis for the in-depth interviews. One interview was held to cover the general business or policy perspective (mainly with CEOs, marketing executives, and general managers) and one to cover the operations and IT perspective (mainly with COOs, CIOs, and CTOs). The average duration of the interview was 90 to 120 minutes. Basis for the interviews were the perceived agility gaps identified by the respondents in the survey. From each interview, minutes were taken and checked for accuracy with the interviewee.

We chose to study four business sectors and three public sectors in the Netherlands:

- Logistics (logistics service providers)

- Finance (retail banking)

- Utilities (distribution and sales of energy)

- Mobile telecom (mobile telecom operators)

- Central government (Dutch ministries)

- Higher education institutes

- Other public sectors (operational authorities such as tax authorities, local authorities, etc.)

These sectors constitute an important segment of the total Dutch business and public sector. Furthermore, these sectors are confronted with a wide variety of external and internal change factors, such as regulations, shifts in customer demands, reorganizations, and changes in IT.

\footnotetext{
${ }^{2} \mathrm{~A}$ copy of the full questionnaire can be obtained from the researchers.
} 
Table 5.1

\begin{tabular}{|c|c|c|c|c|c|}
\hline \multicolumn{6}{|c|}{ Business Agility Change Factor Scores } \\
\hline $\begin{array}{l}\text { Change Factors } \\
\text { Capability 1: Operational }\end{array}$ & \multicolumn{3}{|c|}{$\begin{array}{l}\text { Absolute scores } \\
\text { (Scale from } 1 \text { to } 5 \text { ) }\end{array}$} & \multicolumn{2}{|c|}{$\begin{array}{l}\text { Variance } \\
\text { (Max-min score over } 7 \text { sectors) }\end{array}$} \\
\hline 1) Growing demand for financial transparency and accountability (Basel-2, IAS etc.) & 3.40 & 2.93 & 0.47 & 1.80 & 0.72 \\
\hline 2) New regulation at the national level & 3.49 & 3.00 & 0.49 & 1.30 & 2.00 \\
\hline 5) Increased outsourcing of IT-related systems and personnel* & 3.28 & 2.83 & 0.45 & 0.80 & 1.10 \\
\hline 6) Emerging price war (market focused on price)/shrinking margins & 4.06 & 1.94 & 2.12 & 1.45 & 1.20 \\
\hline 7) Need for lower-priced services & 3.32 & 2.43 & 0.89 & 1.50 & 1.33 \\
\hline $\begin{array}{l}\text { 8) Changing requirements take too long to implement into the organization and } \\
\text { systems* }\end{array}$ & 3.30 & 2.01 & 1.29 & 1.26 & 1.62 \\
\hline 12) Desire to increase the levels of expertise of employees* & 2.59 & 2.12 & 0.47 & 0.67 & 1.17 \\
\hline 13) Reorganization of internal processes* & 3.49 & 2.65 & 0.84 & 0.48 & 0.04 \\
\hline
\end{tabular}




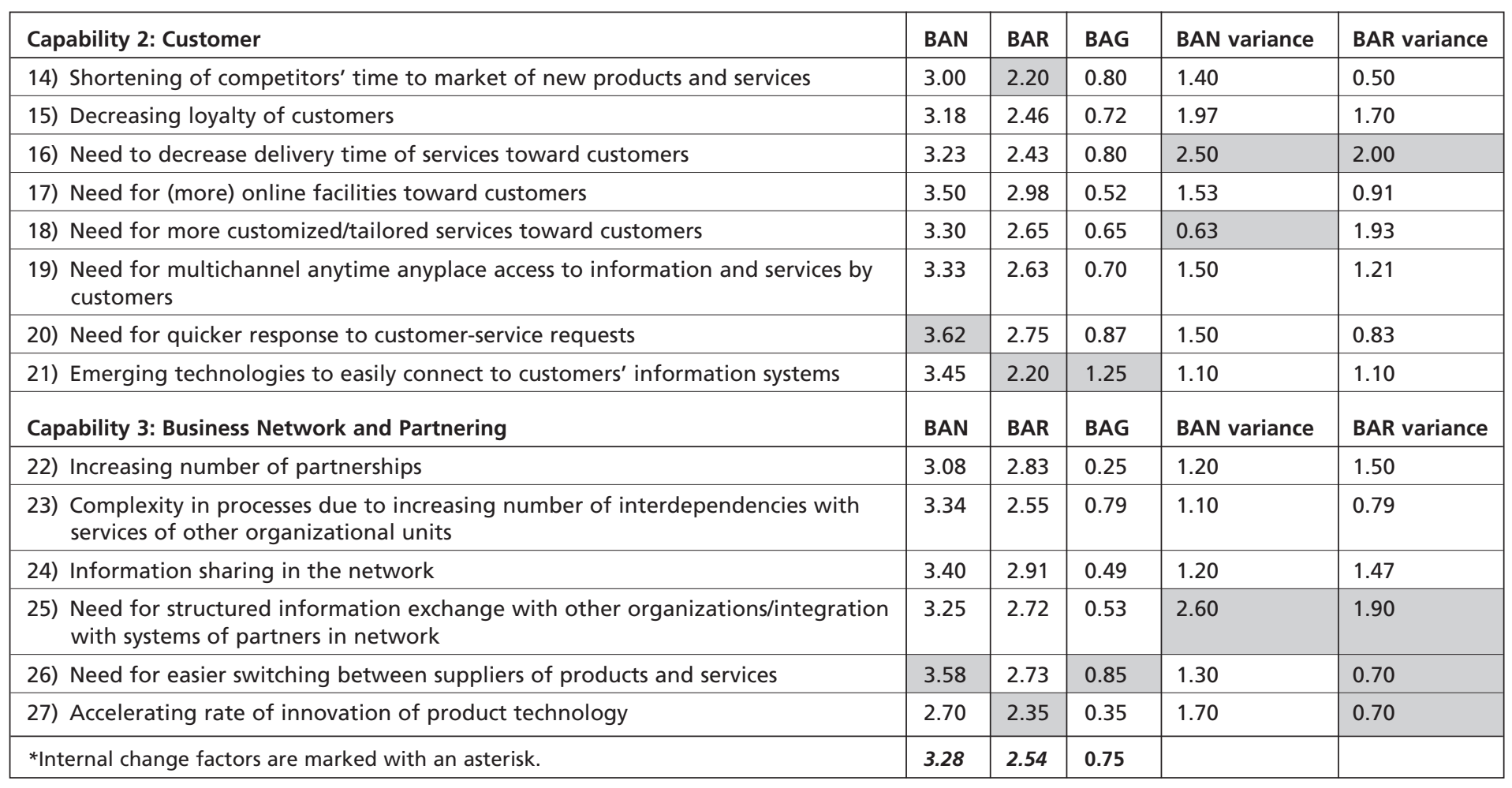




\section{Findings}

We will present three types of findings. First, we will present an overview of the average scores on BAN, BAR, and BAG per sector and per dimension of business agility. Next, we will compare the public sectors with the business sectors on BAN, BAR, and BAG. Finally, we will analyze the importance of individual change factors per dimension of business agility.

\section{Overall Differences between Sectors}

Table 5.2 compares the seven sectors on BAN, BAR, and BAG per dimension of business agility. When we look at the overall BAN scores, logistics has the highest BAN (3.63) on all three dimensions. The lowest BAN is found in the education sector for the operational dimension (3.17), in the energy sector on the customer dimension (2.91), and in the finance sector on the network dimension (2.85).

When we look at the overall BAR scores, the energy sector has the lowest BAR on the operational dimension (2.08) and the customer dimension (1.91), while the lowest BAR is found in the other public sector on the network dimension (2.42). The highest BAR is found in the telecom sector on the operational dimension (2.91), in the government sector on the customer dimension (3.15), and in the logistics sector on the network dimension (3.07).

When we look at the overall BAG scores, the highest scores are found in the logistics (1.08) and energy (0.96) sectors, and on the operational (0.81) and customer (0.79) dimensions. On the operational dimension, the highest BAG scores are found within the logistics (1.33) and energy (1.21) sectors, on the customer dimension in the energy (1.00) and finance (0.96) sectors, and on the network dimension in the other public sector (1.15).

\section{Public versus Business}

When we compare the three public sectors with the four business sectors on BAN, BAR, and BAG, we find a few differences. BAN is about the same within public and business, only BAN on the business network dimension scores higher in public (3.41) compared to business (3.19). BAR is higher in public on the operational and customer dimensions, but slightly lower on the business network dimension. These differences are also found when we compare the BAG scores. Overall BAG scores within public are lower compared to business, with the exception of the average BAG score on the business network dimension, which is considerably larger within public $(0.81)$ compared to business $(0.51)$.

\section{Importance of Individual Change Factors and the Role of IT}

An analysis of the individual change factors will further clarify the differences between the seven sectors on changes which are related to the three dimensions of business agility. Table 5.1 presents the average scores on the 27 IT-related change factors on BAN, BAR, and BAG. Furthermore, we have included the variance between the seven sectors analyzed on BAN and BAR. The change factors have been grouped into the three major business agility capabilities: factors affecting operational agility, factors affecting customer agility, and factors affecting business network and partnering agility. We will now discuss the largest BAGs per agility capability category and the effects of IT on BAR, as found in our survey and discussed during the interviews. We will use examples from the different sectors to illustrate our findings. 
Table 5.2

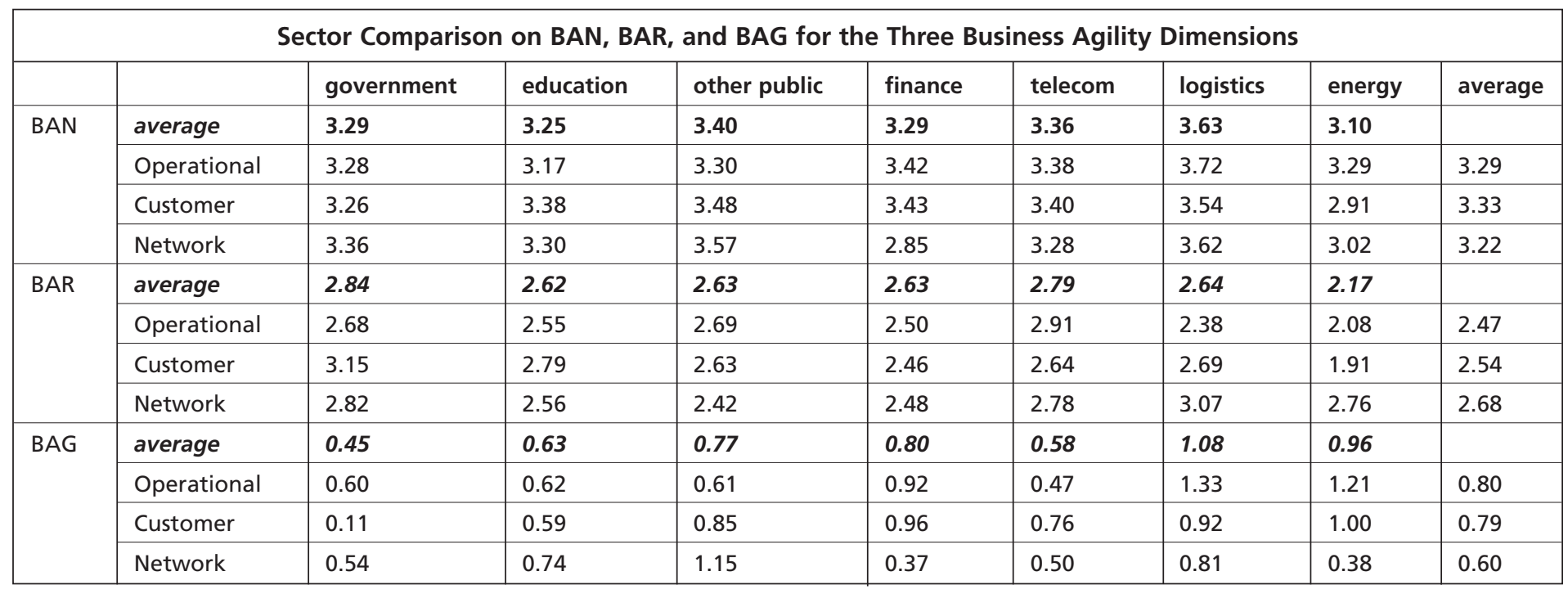




\section{Operational Business Agility}

The change factor with the highest BAG (overall and within the operational dimension; $\mathrm{BAG}=2.12$ ) is the emerging price war and shrinking margins (\#6). This change factor influences all the business sectors analyzed and, to a lower degree, the public sectors. Companies have a lot of difficulties coping with the required changes in their internal processes. Lowering the prices requires changes in operational processes to cut costs as it influences the way companies are structured and operate. This is an important driver for re-organizing the internal processes (\#13) and major organizational change (\#9). Many respondents mentioned the case of mergers and acquisitions as an example of major organizational change, where merging and integrating the various IT infrastructures was most time-consuming and caused the highest gaps.

Some of the deeper reasons behind the agility gaps in the operational agility capability can be found in the fact that implementing changing requirements into the organization and IT systems takes too long (\#8). Many respondents indicated that in many legacy systems business rules are embedded. There is no distinction between, data, applications, and business rules, which hampers BAR. Since increasingly time and money is spent on maintenance and support of the existing IT infrastructure (\#11), insufficient budget remains for investing in innovation and creating options for a more agility-enhancing architecture.

As a solution to the problems described, many organizations are considering or are already active in the outsourcing of IT resources and personnel (\#4 and \#5). In our research, we saw a large variance between the sectors in the perceived BAR to deal with outsourcing (\#4 BAR variance $=2.33$ ). Lowest BAR was found in the other public sector segment $(\# 4$ BAR $=1.67)$, followed by the finance sector $(\# 5$ BAR $=2.10)$. Main reasons for outsourcing are reduction of costs, standardization of the IT infrastructure, and a focus on core competences. Respondents mentioned a number of difficulties involved in outsourcing. Strategic decisions need to be made on the degree of outsourcing. Furthermore, governance of the outsourcing provider creates new transaction costs. If part of the outsourcing deal is based on off-shoring, governance requires dealing with cultural issues and very clear and detailed specifications of change requests. In general, respondents provided both pros and cons for the proposition that outsourcing enhances BAR.

Another important change factor leading to a high BAN is new regulation on national level (\#2) (BAN = 3.49) and specifically, increasing demands from transparency and accountability regulation (\#1) (BAN $=3.40$ ). Financial transparency and accountability causes the highest gap in the finance sector $(B A G=2.20)$. Examples of accountability regulation directly impacting organizations within finance are Basel 2, International Financial Reporting Standards (IFRS), International Accounting Standards (IAS), and Sarbanes Oxley. A lot of organizations within finance have IT systems, organized per product (group). This makes it difficult to comply with the transparency requirements from the new regulations, which are needed on a horizontal level crossing the various products groups.

Executives in all sectors that we studied perceive a high effect-and-response uncertainty with regard to government regulation measures. This leads to high BAN scores. The amount of new regulation, the problem of lack of implementation details, and the timing make it necessary to implement the required changes in a short time frame. This is causing BAGs within the energy (2.00), finance (1.20), and education (1.05) sectors. It is interesting to note that telecom organizations feel that they are overprepared 
(\#2 BAG = -1.3). Apparently, telecom organizations have found ways to deal with uncertainty in regulation.

Some change factors are dependent on the domain (business or public). Within the public sectors, we find two change factors that cause relatively large BAGs. Digitization of documents and the usage of e-signatures (\#10) create BAGs within central government (1.26) and the other public sector (0.96). Digitalization of documents and signatures plays an important role to streamline policy decision making and transactions between citizens and government agencies, but has far-reaching impacts on the whole workflow throughout and between organizations, which explains the low BAR scores. Another BAG we found in all three public sectors (with average BAG $=0.97$ ) is increasing the levels of expertise of employees (\#12). The information society and changing role of the public sector requires other types of expertise. Main factors hindering agility as found during our interviews were the aging workforce, insufficient change-oriented people, and a loss of expertise due to the usage of temporary external expertise, which insufficiently remains anchored in the organization.

\section{Customer Business Agility}

The change factor requiring customer business agility capability with the highest BAG (1.25) is connecting to customers' information systems (\#21). Connecting to customer information systems requires an IT architecture with quick-connect capabilities on the basis of open standards and the usage of middleware. A lot of organizations in our sample were insufficiently ready to handle these required quick-connect capabilities.

The second highest BAG is found for responding quicker to customer service requests $(\# 20)(\mathrm{BAG}=0.87)$. Especially the logistics $(\mathrm{BAG}=1.37)$, energy $(\mathrm{BAG}=$ $1.30)$, and other public $(B A G=1.04)$ sectors are insufficiently ready to deal with this agility need. For many organizations, these are large-scale processes, with many customer service requests. Especially public sector organizations and respondents in the energy sector (former public) need a redesign of their internal processes to become more customer-oriented.

Dealing with shortening of competitors' time to market of new products and services (\#14) causes a BAG within the telecom sector (BAG $=1.30)$. There is a high pressure to bring new products and services onto the market within a short time frame. For instance, the introduction of new mobile payment models, data services, new content concepts based on increased bandwidth, and new location-based services require fundamental changes in the organization's procedures, systems, and partnerships.

Customization of services toward customers (\#18) scores relatively equal on BAN over the seven sectors analyzed. Especially within energy there is a low BAR (BAR = 1.50). This is related to decreasing loyalty of customers (\#15) in the energy sector, which scores high on BAN (4.30) and very low on BAR (1.30). Energy companies have been formed by mergers of various previously state-owned energy companies, which each had their own systems and procedures. Furthermore, inherited systems were never designed with a customer or service customization perspective. However, the open market requires customization to attract new customers or preserve existing customers. In the past, these companies did not have to worry about customers, since they did not have the possibility to switch to a competitor. Now this has changed, and fundamental changes are required into culture, processes, and IT systems.

The highest BAG within the finance sector on the customer dimension is caused by the need for multi-channel any time any place access to information and services 
by customers (\#19) (BAG =1.70). The original IT architectures of large financial institutes insufficiently support adding new channels for communication and transactions. Given the increasing importance of Internet banking and the opportunities of mobile payment, there is a high urgency to change IT architecture and systems to support the Internet and mobile devices as channels for communication and transactions.

\section{Business Network and Partnering Agility}

The highest BAG in the business network dimension is found in the need for easier switching between suppliers of products and services (\#26) (BAG =0.85). Especially within logistics $(B A G=1.5)$ and energy $(B A G=1.1)$, BAR is insufficient. To deal with this need, arrangements need to be made on business network level within the industry sector. In the energy sector, the energy clearinghouse has been set up by a number of energy companies to arrange information exchange on a standardized way to facilitate the switching of customers from one supplier to another. Defining the standards and connecting the different systems caused the most difficulties.

The second highest BAG is the complexity in processes due to increasing number of interdependencies in the business network (\#23). This change factor scores relatively high on BAN (3.34) and low on BAR (1.55). Especially respondents within the education $(\mathrm{BAG}=1.30)$, other public $(\mathrm{BAG}=1.34)$, and energy $(\mathrm{BAG}=1.10)$ sectors find themselves insufficiently ready for this business network integration. This factor is closely related to information sharing in the network (\#24). Increasingly, public services are interdependent, and information needs to be shared between different organizations in the public sector. Public services more and more will make use of authentic registers, whereby distributed databases need to be coupled to provide a complete information profile on citizens for various types of services. Furthermore, government is working on a single portal for governmental services, a single window between citizens and the government for information exchange, information access, and services. In the back office, this means a lot of distributed databases need to be connected, which creates a lot of interdependencies.

The need for structured information exchange (\#25)-think about EDI and XML — causes a high variety in BAN and BAR between the seven sectors analyzed. Highest BAG scores are found in logistics $(\mathrm{BAG}=2.03)$ and other public $(\mathrm{BAG}=0.94)$ sectors. In the logistics sector, the need for chain-wide tracking and tracing requires integration to partner information systems. Given the diversity in type of companies and size (a lot of Small-to-Medium Sized Enterprises (SMEs)), it is difficult to achieve chain-wide structured information exchange.

The lowest BAR is found in managing the accelerating of innovation of product technology (\#27) (BAR = 1.35). Telecom companies are highly dependent on their mobile device and content partners to use this change factor as a way to innovate and distinguish from competitors. Especially within telecom, this causes a BAG (BAG = 1.2). New technologies in mobile devices; voice-over IP; emerging data services; and the merging of phone, Internet, and TV have resulted in a series of innovations in product technology. Customized products and services need to be put on the telecom market in ever shorter time.

\section{Analyses}

Figure 5.2 shows a plot of the relative scores on perceived BAN and perceived BAR for the 27 change factors analyzed. First, we calculated the overall average scores on BAN (3.28) and BAR (2.54). We then related the individual scores of the 27 change 


\section{Business Agility Change Factors Matrix}

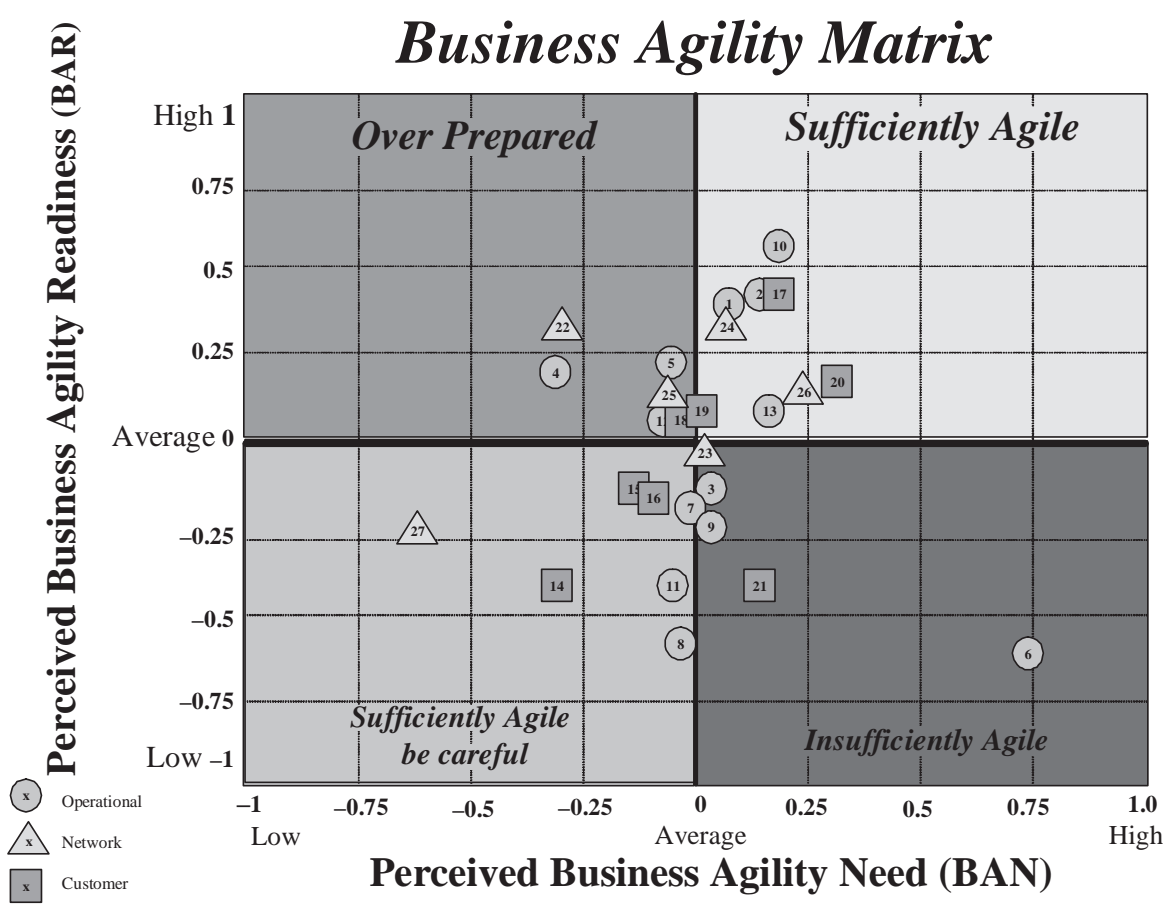

factors to this overall average score by subtracting the overall average score from the individual score. This way, we made the relative importance and relative scores of the 27 change factors on BAN and BAR explicit.

Based on their relative position on the Business Agility Matrix, factors can lead to a BAG. If BAN scores above average, and BAR below average, organizations are insufficiently agile and need immediate action to deal with the change factor. Most important change factors requiring immediate action as found in our research were emerging price war and shrinking margins (\#6), followed by connecting to customers' information systems (\#21) and dealing with major organizational change (\#9). A number of change factors need a careful watch. If the level of change increases, they also require immediate action. If BAN is below average and BAR is above average, organizations are more or less overprepared to deal with a change factor.

It is interesting to note that respondents find that their BAR to deal with change factors related to business network agility is relatively sufficient, compared to other factors. This can be explained by the fact that more attention is given to the internal operational business agility. Either there is yet insufficient awareness on the importance of the business network perspective, or solving internal problems still has priority over business network opportunities, or the business network is not found to be important. 
Since Figure 5.2 only provides an overview of average scores over the total sample, the business agility matrix will look different per sector analyzed. As an example, we will show the business agility matrices for central government and finance (Figure 5.3).

Relative importance of change factors on a sector level becomes more explicit, with a wider range of scores on BAN and BAR. Given the differences between different sectors on the relative scores for change factors on BAN and BAR, sector-specific benchmarks are needed for organizations to assess and compare their BAN and BAR scores on various change factors.

This study shows that some change factors are generic, but some are dependent on public or private domains. These factors have a relatively similar type of BAN for all business sectors (e.g., \#6) or for all public sectors (e.g., \#10, \#12, and \#24). Furthermore, various change factors are sector-specific (e.g., \#17 and \#27). BAR in general is organization-specific, although the same types of challenges in increasing business agility IT capabilities are found in all organizations analyzed. Depending on the position of a change factor in the Business Agility Matrix, several IT strategies can be defined (Figure 5.4).

If an organization finds itself in the Insufficiently Agile quadrant for a certain change factor, two possible IT strategies can be used. In the first strategy, IT is used to increase the BAR. This includes increasing both the sensing and respond capabilities (Dove, 2001). A few general guidelines for increasing BAR were extracted from our interviews and confirmed in the literature. Respondents believe that IT architecture and standards should be managed centrally at enterprise level on the basis of a broadly enforced set of technology standards, while keeping room for local responsiveness. To some degree, the same accounts for security and risk and IT facilities management. This was also recommended by Weill, Subramani, and Broadbent (2002). Secondly, an infrastructure that is modular, service-based, and tailored to the enterprise's strategy (close alignment between business and IT) is expected to enhance business agility. Such an architecture is loosely coupled, based on modular reusable components in a scalable framework (Dove, 2001). A distinction between data, applications, and business rules creates the basis for more agility. Compatibility and integration can be achieved via standardized interfaces and connections, usage of standardized technology to store data (such as XML), and usage of interoperability and integration supporting standards and open protocols (e.g., XML and Web services) (Vervest and Dunn, 2000; Brown and Bessant, 2003). These are enablers for increasing the business network agility capability (van Hillegersberg, et al., 2005) as well as the internal operational business agility capability. A final guideline we found in the literature (Weill, Subramani, and Broadbent, 2002) is that the organization's infrastructure should be created via a series of incremental investments. Staged investment means partitioning a larger IT investment into stand-alone increments that build on the preceding ones, thereby creating strategic real options (Konsynski and Tiwana, 2004).

In the second strategy, IT is used to decrease the BAN. As an example, we take the need to respond more quickly to customer service requests (\#20). An IT strategy to lower BAN might be to create self-service environments where customers can help themselves or each other, based on access to their data, transactions, and intelligent decision support tools for solving problems. This can be extended to self-service environments, where customers are offered personalized products and services based on real-time product configurators and historic databases that match profiles to offers (like Amazon). By creating self-service environments, there will be less customer 
Figure 5.3

Business Agility Change Factors Matrices: Central Government and Finance
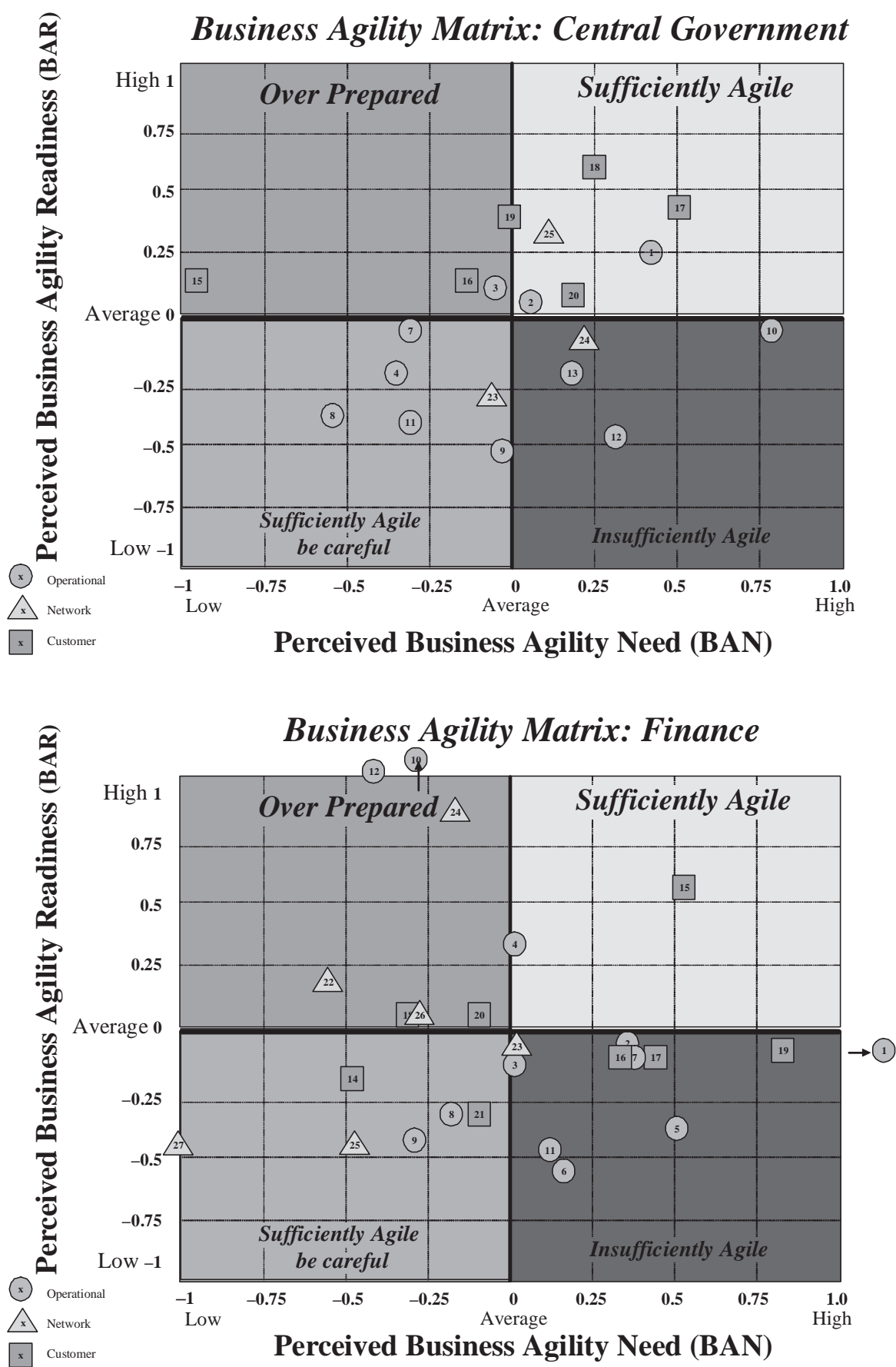


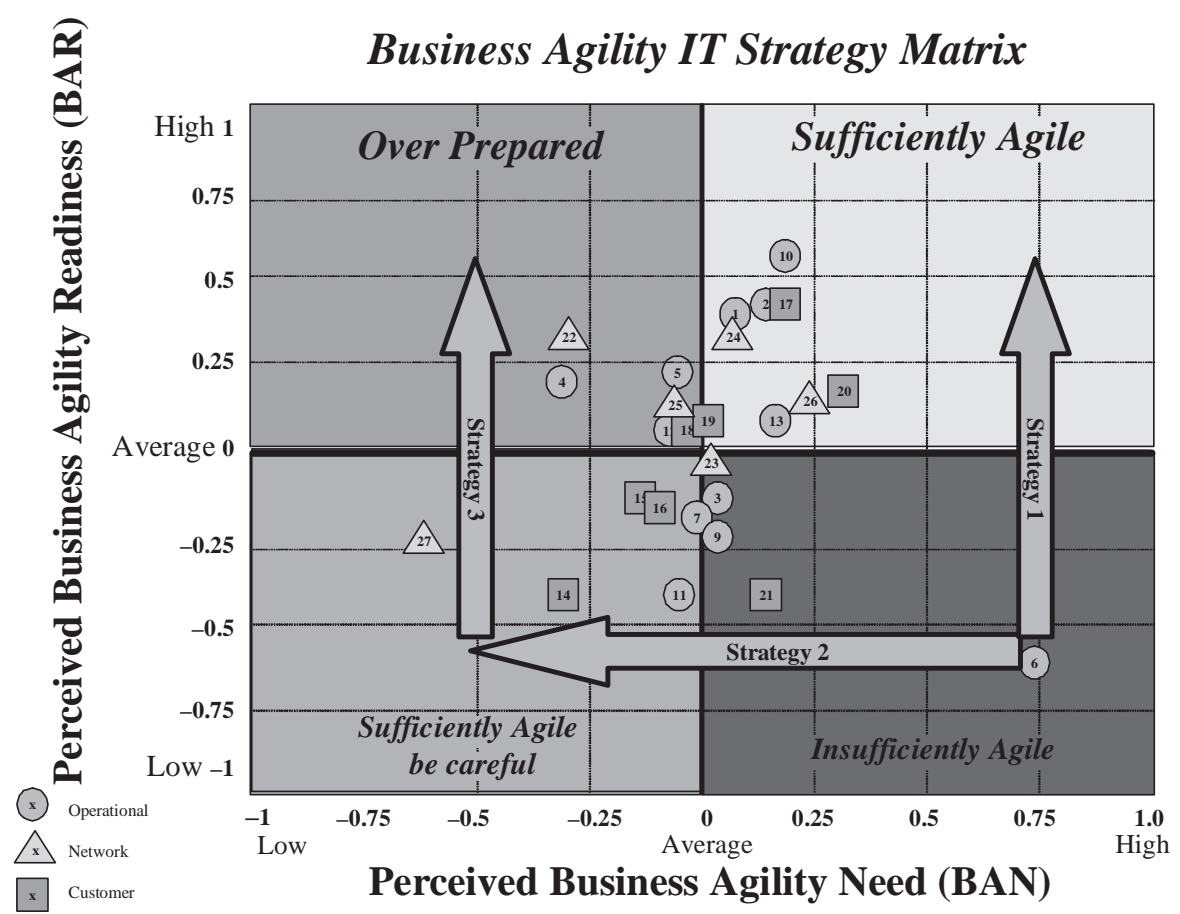

service requests and therefore the BAN score of the need to quickly respond to customer service requests will decrease.

If an organization finds itself in the Sufficiently Agile, Be Careful quadrant for a certain change factor, the IT strategy should focus on increasing the sensing capabilities. Sensing can be achieved by early detection systems, which alert at the first signs of a new threat or opportunity with procedures to determine a proper response (Daft et al., 1988; Conner, 2000). The involvement of customers in product developmentso-called customer sensitivity—can also be an important basis for sensing (Maskell, 2001; Van Hoek et al, 2001). Available, complete, pertinent, and easy-to-access information on customer needs, anxieties, and service requirements via IT is a key enabler for agility (Christopher, 1992).

\section{Conclusion}

The central research question of this chapter was: What are the contributing factors that require business agility, and what IT strategies can be implemented for enhancing business agility? First we defined the concept of business agility. Our definition is "Business agility is the ability to sense highly uncertain external and internal changes and respond reactively or proactively, based on innovation of the internal operational processes, involving the customer in exploration and exploitation while leveraging the capabilities of partners in the business network." 
This study shows that some change factors are generic, but some are dependent on public or private domains. The emerging price war and the need for lower priced products and services combined with fast-changing customer requests dramatically influence all business sectors analyzed. Companies face severe difficulties in coping with the required changes. In many cases, this requires a totally different way of organizing the company and its business network. Additionally, new regulation is causing high BAN in almost all sectors analyzed. Furthermore, various change factors are sector-specific. Given the differences between different sectors on the relative scores for change factors on BAN and BAR, sector-specific benchmarks are needed for organizations to assess and compare their BAN and BAR scores on various change factors.

The results also indicate that BAN is not just created by uncertainty about external changes. Many internal changes (such as mergers and acquisitions, changes in systems and procedures, digitalization of documents, and e-signatures) require organizations to increase their BAR scores. This is reflected in the BAG scores we found for various change factors with a more internal origin in different sectors.

BAR in general is organization-specific, although the same types of challenges in increasing business agility IT capabilities are found in all organizations analyzed. Respondents are very worried about the pace at which responses to the changes can be implemented. To a large degree, this can be explained by the existing organizational structures, cultures, and legacy infrastructures.

The highest BAG scores were found in logistics and energy, while changes requiring agility on the operational and the customer dimension caused larger gaps than the business network dimension. The highest BAG scores on the operational dimension were found within logistics and energy, on the customer dimension in energy and finance, and on the business network dimension in other public arenas.

Depending on the position of a change factor in the Business Agility Matrix, three generic IT strategies were defined. If an organization finds itself in the Insufficiently Agile quadrant for a certain change factor, two possible IT strategies can be used. In the first strategy, IT is used to increase the BAR. This includes increasing both the sensing and respond capabilities (Dove, 2001). In the second strategy, IT is used to decrease the BAN. If an organization finds itself in the Sufficiently Agile, Be Careful quadrant for a certain change factor, the IT strategy should focus on increasing the sensing capabilities.

\section{References}

Brown, S. and Bessant, J. (2003). The manufacturing strategy-capabilities links in mass customization and agile manufacturing: An exploratory study. International Journal of Operations \& Production Management, 23 (7-8), 707-730.

Canter, J. (2000). An agility-based OODA model for the e-commercele-business enterprise. Retrieved from: http://www.belisarius.com/modern_business_strategy/canter/canter.htm.

Christopher, M. (1992). Logistics \& Supply Chain Management: Strategies for Reducing Cost and Improving Service. London, UK: Pitman.

Conner, D. R. (2000). How to create a nimble organization. National Productivity Review, 19 (4), 69-74.

Conboy, K. B., Fitzgerald. (2004). Towards a conceptual framework of agile methods: A study of agility in different disciplines. In Proceedings of the 2004 ACM workshop on interdisciplinary software engineering research. Newport Beach, CA, pp. 37-44.

D'aveni, R., Richard A., Gunther, R. E. (1994). Hypercompetition: Managing the Dynamics of Strategic Maneuvering. New York: The Free Press. 
Daft, R. L., Sormunen, J., and Parks, D. (1988). Chief executive scanning, environmental characteristics, and company performance: An empirical study. Strategic Management Journal, 9 (2), 123-139.

Dove, R. (2001). Response Ability: The Language, Structure and Culture of the Agile Enterprise. New York: Wiley.

Goldman, S., et al. (1991). 21st Century Manufacturing Enterprise Strategy. Bethlehem, PA: Iacocca Institute, Lehigh University.

Goldman, S., Nagel, R., and Preiss, K. (1995). Agile Competitors and Virtual Organizations. New York: Van Nostrand Reinhold.

Hooper, M. J., Steeple, D., and Winters, C. N. (2001). Costing customer value: An approach for the agile enterprise. International Journal of Operations and Production Management, 21 (5), 630-644.

Kidd, P. T. (1994). Agile Manufacturing: Forging New Frontiers. Wokingham, UK: AddisonWesley.

Kidd, P. T. (1995). Agile Corporations: Business Enterprises in the 21st Century-An Executive Guide. Macclesfield, UK: Cheshire Henbury.

Konsynski, B. and Tiwana, A. (2004). The improvisation-efficiency paradox in inter-firm electronic networks: Governance and architecture considerations. Journal of Information Technology, 19 (4), 234-243.

Maskell, B. (2001). The age of agile manufacturing. Supply Chain Management: An International Journal, 6 (1), 5-11.

Mason-Jones, R. and Towill, D. R. (1999). Total cycle time compression and the agile supply chain. International Journal of Production Economics, Special Issue 62 (1-2), 61-73.

Milliken, F. J. (1987). Three types of perceived uncertainty about environment: State, effect, and response uncertainty. Academy of Management Review. 12 (1), 133-143.

Preiss, K., Goldman, S. L., and Nagel, R. N. (1996). Cooperate to compete: Building agile business relationships. New York: Van Nostrand Reinhold.

Ramasesh, R., Kulkarni, S., and Jayakumar, M. (2001). Agility in manufacturing systems: An exploratory modeling framework and simulation. Integrated Manufacturing Systems, 12 (7), 534-548.

Sambamurthy, V., Bharadwaj, A., and Grover, V. (2003). Shaping agility through digital options: Reconceptualizing the role of information technology in contemporary firms. MIS Quarterly, 27(2), 237-263.

Schrage, M. (2004). The struggle to define agility: It's all about the Execution. CIO Magazine, Aug. 15th, 2004 . Retrieved from: http://www.cio.com/archive/081504/schrage. html.

Sharifi, H. and Zhang, Z. (1999). A methodology for achieving agility in manufacturing organizations: An introduction. International Journal of Production Economics, 62 (1-2), $7-22$.

Swafford, P. M. (2003). Theoretical development and empirical investigation of supply chain agility. Dissertation, Georgia Institute of Technology, Atlanta, April, pp. 1-138.

van Hillegersberg, J., Boeke, R., and Van den Heuvel, W. J. (2005). The potential of web services to enable Smart Business Networks. In Smart Business Networks (P. H. M. Vervest, E. V. Heck, K. Preiss, L. F. Pau, eds.). Berlin-Heidelberg: Springer-Verlag, pp. 349-362.

van Hoek, R. I., Harrison, A., and Christopher, M. (2001). Measuring agile capabilities in the supply chain. International Journal of Operations \& Production Management, 21 (1/2), 126-147.

Vervest, P. and Dunn, A. (2000). How to Win Customers in the Digital World: Total Action or Fatal Inaction. Berlin-Heidelberg: Springer-Verlag.

Vervest, P. H. M., Heck, E. V., Preiss, K., and Pau, L. F. (eds.) (2005). Smart Business Networks. Berlin-Heidelberg: Springer-Verlag.

Wadhwa, S. and Rao, K. S. (2003). Flexibility and agility for enterprise synchronization: Knowledge and innovation management towards flexagility. Studies in Informatics and Control, 12 (2), 111-128. 
Weill, P., Subramani, M., and Broadbent, M. (2002). IT Infrastructure for Strategic Agility. MIT CISR Working Paper, No. 329, Center for Information Systems Research, Sloan School of Management.

Yusuf, Y. Y., et al. (2004). Agile supply chain capabilities: Determinants of competitive objectives. European Journal of Operational Research, 159 (2), 379-392.

Zhang, Z. and Sharifi, H. (2000). A methodology for achieving agility in manufacturing organizations. International Journal of Operations \& Production Management, 20 (4), 496-513. 